\title{
Intralesional Injection of Bleomycin in Verruca Vulgaris Immunohistochemical and Clinical Study
}

\author{
E.M.Akl ${ }^{1}$, A.A.Saleh ${ }^{1}$, H.Rashad ${ }^{2}$ and M.S.Hakem ${ }^{1}$ \\ ${ }^{1}$ Dermatology and Venerology Dept., Faculty of Medicine, Benha Univ., Benha, Egypt \\ ${ }^{2}$ Pathology Dept., Faculty of Medicine, Benha Univ., Benha, Egypt \\ E-Mail:mareez@gmail.com
}

\begin{abstract}
The current examination was an endeavor to evaluate the viability of IL bleomycin infusion for the treatment of verruca vulgaris. Just as to evaluate the results that were experienced during the investigation. This investigation was done on 30 patients grumbling of various verrucae vulgaris. Included 10 ordinary people, biopsy was taken from non sun uncovered skin (lower arm) and recolored by caspse3. 15 of them were biopsied before infusion of bleomycin intralesionally and inspected histologically and immuno-histochemiclly by H\&E and caspase 3 . similar 15 patients with another verrucae were biopsied after infusion of bleomycin intralesionally in third ,fourth and seventh day after infusion and analyzed by $\mathrm{H} \& \mathrm{E}$ and caspase 3 . the remainder of verrucae from all patients were infused bleomycin intralesionally like clockwork for 6 meetings most extreme and followed for complete leeway and repeat as long as 3 months. there was factually centrality in articulation and force of caspase 3 shows expanding in apoptosis after infusion, the articulation was cytoplasmic in dispersion and sketchy in design in all cases. intralesional bleomycin is a powerful and very much endured first line treatment in the treatment of verrucae vulgaris paying little mind to patient's age, sex, malady span or being treated before by any modalities.
\end{abstract}

\section{Introduction}

Verruca vulgaris (VV) are one of the most wellknown viral diseases influencing the skin. It is brought about by the human papilloma-infection (HPV). verrucae vulgaris present as firm keratotic papules that can show up on any cutaneous surface and mucosa [1].

Verrucae vulgaris are treated with various physical modalities, for example, electro-medical procedure, cryosurgery, and diverse physical or substance attacks with variable outcomes [2].

High paces of repeat or scarring are accounted for in every one of these modalities. Intralesional bleomycin is recommended to be potential treatment methodology in treating moles and has been utilized since the 1970s in western nations. Bleomycin is a cytotoxic glycopeptide anti-toxin secluded from a strain of microorganisms Streptomyces verticillus [3].

Bleomycin has antitumor, antibacterial, and antiviral exercises which might be identified with its capacity to tie with deoxyribonucleic corrosive (DNA), causing DNA strand scission, and disposal of pyrimidine and purine bases [4].

Bleomycin doesn't tie legitimately to HPV. The plausible system of activity of bleomycin is by influencing cell DNA union; and furthermore by controlling keratinocyte turnover in this manner influencing viral endurance [5].

Intralesional bleomycin infusion successful in treatment of moles either by infusion into the injury by needle or by utilizing dermojet or prick technique [6].

Caspase-3 is a significant killer caspase that is severed and actuated by both caspase- 8 and caspase- 9 initiator caspases. Caspase- 3 is cut at an aspartate buildup to yield a p12 and a p17 subunit toform the dynamic caspase-3 compound. Dynamic caspase-3 debases different cell proteins and is liable for morphological changes and DNA discontinuity in cells during apoptosis [7].
Apoptosis is a significant instrument through which the host can kill tainted cells, and the capacity to dodge apoptosis can accordingly upgrade infection endurance. Apoptosis assumes a significant part during keratinocyte separation. Therefore, it is to the HPV preferred position to intercede in apoptotic pathways, and five papillomavirus proteins, E2, E5, E6, E6* and E7, have been appeared to do as such [8].

The point of this examination is to assess the adequacy of intralesional infusion of bleomycin in the treatment of verruca vulgaris, and to survey its impact on Caspase 3 articulation.

\section{Patient and method}

Prospective case control study was done in Dermatology \& Andrology and Pathology Departments in Benha University .

Approval of the Dermatology and Andrology Department and the Ethics Committee in the Faculty of Medicine, Benha University has been taken before preceding the study.

This study included 30 participants complaining from multiple verrucae vulgaris, were injected bleomycin, have been selected from Benha University Hospitals.

\subsection{All subjects will be subjected to the following}

1- All patients were complaining of verruca vulgaris.

2- The size of warts was more than $0.5 \mathrm{~cm}$ in order to have good biopsies after injection of bleomycin.

3- Full medical history taking from the patients .

4- Immunohistochemistry: Caspaase 3 expression will be detected by immunohistochemistry.

5- Age more than 18 years old.

\subsection{Exclusion criteria for patients}

1- Pregnancy, hepatits or, renal diseased patients and patients under chemotherapy. 
2- Patients with Raynaud's phenomenon or vascular diseases

3- Patients who have been treated from verruca vulgaris in the last 3 months.

4- Hypersensitivity to bleomycin.

5- Blood diseases.

6- Patients refused biopsy

7- Connective tissue disease, rheumatoid artharitis

\subsection{They divided into 2 groups}

- Group (A): As a control group of 10 healthy volunteers were biopsied from normal non sun exposed skin using 4-6mm punch biopsies.

- Group (B):20 patients with multiple verruca vulgaris subjected to

- Group (B I): 15 of them verrucae vulgaris were biopsied before injection of bleomycin for histological and immunohistochemical examination.

- Group (B II): the same 15 with another verrucae vulgaris were biopsied after injection of bleomycin after 3,4 and 7days for histological and immunohistochemical examination

\subsection{Materials used}

- 15 unit Bleomycin sulfate vial as lyophilized powder reconstituted by $10-\mathrm{ml}$ sterile physiologic saline solution manfectured by CELON LABS. Part of this solution was injected intralesionally into verruca valgaris till bleaching effect with maximum dose $5 \mathrm{Iu}$

- Two disposable tuberculin syringes $(1 \mathrm{ml})$ fitted with a 30-gauge needle (built-in needle)

- Isopropyl alcohol as disinfectant to warts and adjacent skin.

- Ethyl chloride spray as local anesthetic before injection of bleomycin.

- Mepevacaine $\mathrm{HCl} 2 \%$ was used as infiltration anesthetic before punch biopsies were taken.

- Disposable 4-6 mm punch

- The biopsies were taken before bleomycin injection and after, 3th, $4^{\text {th }}$ and $7^{\text {th }}$ of bleomycin injection. These biopsies were prepared for histological and immunohistochemical examination.

- Group (BIII): the rest of verrucae vulgaris were injected bleomycin and followed clinically up to 3 months for clearance of verruca vulgaris and recurrence.

- Histological and immunohistochemical examination of group IIa and IIb.

Skin biopsies were taken, fixed in $10 \%$ neutral buffered formalin, routinely processed and paraffin embedded blocks were prepared. Sections were mounted on plain slides and routinely stained with hematoxylin and eosin (H\&E) and examined by light microscopy to:

- Confirm the clinical diagnosis of warts in group B

- Detect the effect of intralesional injection of bleomycin into warts. The changes were graded as follows:
- Grade (0): no changes as seen in classic histopathologic picture of verruca vulgaris.

- Grade (1): ischemic necrosis in keratinocytes, hemorrhagic and neutrophilic infiltrate in only one third of verruca vulgaris thickness.

- Grade (2): the same changes in two thirds of verruca vulgaris thickness.

- Grade (3): the same changes in full thickness of the verruca vulgaris.

Adjacent sections were mounted on positive (+ve) charged slides and were subjected to immunohistochemical (IHC) stain for detection of expression of caspase 3 .

\subsection{Clinical assessment of therapy by}

1- Regression of size and area of verruca valgaris .

2- Necrosis

3- Interpretation of IHC results: Immunohistochemically, caspase-3 expression was confirmed by cytoplasmic and/or nuclear stain in examined cells .For every antibody, the following items were assessed:

The epidermis of normal skin biopsies, and verrucae were assessed for:

1- Expression: positive or negative.

2- Intensity: Intensity of expression categorized into 3 groups mild, moderate and strong.

3- Distribution: patchy or diffuse.

4- Pattern: cytoplasmic or nucleocytoplasmic.

5- For Immunohistochemical studies caspas3 antibody staining were performed for all biopsies using Avidin Biotin complex technique

6- For histopathological analysis, sections of $4 \mu \mathrm{m}$ thickness were cut, dewaxed in xylene and then rehydrated in descending grades of alcohol and stained by conventional hematoxylin and eosin (H\& E) stain.

\section{Results}

On considering their qualities; their age went somewhere in the range of 18 and 53 years old with the middle age was $32 \pm 9.06$ years. Female sex was the prevalent sexual orientation $(60 \%)$ and $75 \%$ of them live in metropolitan territory (Zagazig). $60 \%$ of them have various sorts of occupations while others are jobless. Just a single patient is smoker. Two of them had already hand verrouca went on for 1 year and 6 years and were treated by electrocautry.

Three of them had first degree relative with this pathology. On contemplating the clinical models of the injuries introduced in patients; a large portion of the patients $(75 \%)$ had five or less sores which generally influenced the upper appendage $(75 \%)$ and the hand area by name $(65 \%$ of the $75 \%)$. Generally $(65 \%)$ of the experienced size was $0.5 \mathrm{~cm}$ or less and dated for a very long time or less in many patients $(80 \%) .70 \%$ of the patients had no past treatment for it while the most utilized treatment methodology in the individuals who had past treatment was electrocautry $(25 \%)$. 
$60 \%$ of the patients required 3-4 bleomycin infusion settings. In any case, the entirety of the 20 patients experienced improvement as complete fix in all cases aside from in one case indicated decrease in size by $50 \%$ as it were. $80 \%$ of them encountered mellow torment for a normal of three days after infusion. All the patients aside from one had shading changes after infusion generally $(65 \%)$ grayish discolouration. Just $20 \%$ of the patients had post-infusion discharge as a complexity with no experienced bullae arrangement. Concerning just 2 cases repeated following 3 months in both while remaining cases indicated no repeat in the time of development.

After infusion of bleomycin, the level of articulation of caspase 3 in the unhealthy gathering indicated stamped critical distinction when contrasted with a gathering of solid people.
After infusion of bleomycin, all the patients demonstrated articulation of caspase 3 in huge way (minuscule assessment was accomplished for 15 cases as it were).

After infusion of bleomycin, the level of power of caspase 3 expanded in critical way, and the greater part of them indicated moderate level of force (minuscule assessment was accomplished for 15 cases only).all cases were cytoplasmic in appropriation and sketchy in design

Before infusion of bleomycin, the level of articulation of caspase 3 in the sick gathering demonstrated no noteworthy contrast when contrasted with a gathering of sound people.

Being infused with bleomycin; infinitesimal assessment was accomplished for 15 cases just indicating noteworthy changes in both keratinocytes and neutrophil invasions and the most normally experienced evaluation in the two was the first grade.

Table (1) Shows expression of caspase 3 before and after injection of bleomycin injection.

\begin{tabular}{lcccc}
\hline & Before & After & X2 & p-value \\
\hline Yes & 10 & 15 & & \\
No & 5 & 0 & 4.658 & 0.031 \\
Total & 15 & 15 & & \\
\hline
\end{tabular}

Table (2) Shows degree of intensity of caspase 3 before and after injection of bleomycin injection.

\begin{tabular}{lcccc}
\hline & Before & After & X2 & p-value \\
\hline Zero & 5 & 0 & & \\
Mild & 10 & 5 & 8.192 & 0.042 \\
Moderate & 0 & 8 & & \\
Strong & 0 & 2 & & \\
Total & 15 & 15 & & \\
\hline
\end{tabular}

Table (3) Shows degree of expression of caspase 3 in the healthy (control) group and the diseased group before injection.

\begin{tabular}{lcccc}
\hline & Control & $\begin{array}{c}\text { Diseased } \\
\text { before injection }\end{array}$ & X2 & p-value \\
\hline Zero & 2 & 5 & & \\
Mild & 8 & 10 & 0.529 & 0.466 \\
Total & 10 & 15 & & \\
\hline
\end{tabular}

Table (4) Shows degree of expression of caspase 3 in the healthy (control) group and the diseased group after injection.

\begin{tabular}{lcccc}
\hline & Control & $\begin{array}{c}\text { Diseased } \\
\text { after injection }\end{array}$ & X2 & p-value \\
\hline Zero & 2 & 0 & & \\
Mild & 8 & 5 & 12.17 & 0.007 \\
Moderate & 0 & 8 & 9 & \\
Strong & 0 & 2 & & \\
Total & 10 & 15 & & \\
\hline
\end{tabular}

\section{Discussion}

The current investigation was an endeavor to survey the viability of IL bleomycin infusion for the treatment of verruca vulgaris. Just as to evaluate the results that were experienced during the examination and this investigation was performed on 20 patients (bunch BIII) having regular moles were infused $0.1 \mathrm{ml}$ at the base of verruca vulgaris $(0.5 \mathrm{~cm}$ or less up to $1.5 \mathrm{~cm}), 60 \%$ of the patients required 3-4 bleomycin infusion settings. However, the entirety of the 20 patients experienced improvement as complete fix in all cases aside from in one case indicated decrease in size by $50 \%$ as it were, 
In M.D.Saber [9] bleomycin infusion was found exceptionally compelling with fix pace of normal moles was $93.5 \%$ (32 of 34), in anthor study M.I.El-Ghareeb [10] demonstrated $44 \%$ clinical complete reaction of the moles in the 3 meetings in bleomycin gathering. Another examinations indicated $94.9 \%$ complete leeway rate in 39 mole patients treated with bleomycin [11].

In Mahesh [12] the general freedom pace of verruca vulgaris with IL bleomycin was $93.1 \%$, Out of completely cleared verruca vulgaris, $64.19 \%$ verruca vulgaris were cleared with single IL infusion of bleomycin and verruca vulgaris after second IL infusion of bleomycin $35.8 \%$.

Contrasting with results in intralesional $5 \mathrm{FU}$ in verruca in an investigation K.Tahir [13] Overall 30 (75\%) patients (of a wide range of verruca vulgaris) indicated phenomenal outcomes (most extreme six infusions) and stayed clear on development following 2 months of last treatment meeting. Confined aggravation and agony were the significant results experienced in all patients. in any case, in infusion of nutrient D3 intralesional the fix rate was 77.77 (14), in verrucae vulgris in 4 meetings, no torment except for just expanding and dyspigmentation and no recurrance so intralesional bleomycin is preferable in fix rate over 5 FU and vit D3 yet the equivalent in unfriendly impacts like agony with 5FU, vit D3 and 5FU is better in repeat rate in multi month follow up.

The remedial reaction was not influenced by age or sex of patients. Furthermore, was not influenced by sickness span or previous history of other therapy modalities

In this investigation $80 \%$ of patients experienced mellow agony for a normal of three days after infusion. All the patients aside from one had shading changes after infusion generally $(65 \%)$ grayish discolouration. Just 20 $\%$ of the patients had post-infusion discharge as an entanglement with no experienced bullae arrangement, in (10) $60 \%$ of patients were infused bleomycin whined of torment at infusion site that died down 24 hours after infusion. This result that was quickly died down in view of the blend of nearby sedation with bleomycin.

In K. Laura [15], there was roughly $70 \%$ of patients had torment that kept going under 2 days after treatment. In any case, they referenced opposite results as keloid, bullae, Raynaud's wonder that were not revealed in this investigation.

In M.D.Saber [9], the event of limited torment during and after bleomycin infusion was accounted for by essentially all patients. This agony was variable from mellow, moderate to serious and just a single patient created hemorrhagic bullae in two of his infused moles.

In Mahesh [12] Mild to direct agony was the fundamental issue experienced by patients during strategy, for which expansion of bupivacaine hydrochloride $(0.5 \%)$ to bleomycin arrangement assists with mitigating torment, No serious unfavorable impacts were noted aside from hemorrhagic eschar (ecchymosis) was seen in a large portion of the patients with belomycin infusion, which relapsed with hyperpigmentation, hypopigmentation, or insignificant scarring.

In this examination with respect to repeat just 2 cases repeated following 3 months in both while remaining cases demonstrated no repeat in the time of development, however in Mahesh [12]. A sum of $13(16.04 \%)$ out of 81 cleared moles showed repeat during follow up.

Bleomycin is hence an extremely compelling and all around endured first line treatment in the treatment of verruca vulgaris paying little mind to patient's age, sex, illness term or being treated before by any treatment methodology.

In the current investigation we have attempted to explain the potential systems by which bleomycin produces its helpful impacts. 15 patients (bunch BI and BII) griped of verruca vulgaris were exposed to intralesional bleomycin infusion then their verruca vulgaris were biopsied when infusion at various timeframes. These biopsies were exposed to histopathological assessment by customary microscopy in the wake of recoloring by $(\mathrm{H} \& \mathrm{E})$ stain, After bleomycin infusion, practically all verruca vulgaris biopsies indicated histopathological changes as keratinocyte rot $(\mathrm{p}=0.0006)$ and neutrophilic invade $(\mathrm{p}=0.0006)$. These progressions start to show up in verruca vulgaris following 2 days of infusion, it run from grade I.II and III (gentle, moderate to serious). In M.D.Saber [9], all verruca vulgaris biopsies demonstrated huge drain, serious neutrophilic penetrate and liquefactive corruption of keratinocytes.

Modified cell passing assumes a function in the homeostasis of the ordinary epidermis just as in the terminal separation of keratinocytes bringing about a cornified layer shaped by dead keratinocytes that is at last shed from the skin surface. Cysteine proteases that divide their objective proteins at explicit aspartic acids (caspases) assume a significant function in apoptosis. They are available in the cells as idle zymogens that must be cut to create free synergist subunits ready to partner and structure dynamic heterotetramers. The group of caspases can be isolated into two practical subgroups, the initiator and the killer caspases, and caspase-3 is considered similar to a significant killer caspase [16].

\section{References}

[1] P.B.Sheth. Topical and intrlesional antivirl agents. In Wolverton SE, editor. 2nd ed. Comprehensive Dermatologic Drug herapy.Philadelphia, PA: W.B. Saunders Company,Vol.5, PP.576-8.2007.

[2] S.Gibbs. The trials of treating warts. Indian $\mathbf{J}$ Dermatol Venereol Leprol, Vol.80, PP.4956,2014

[3] D.R.Lowy, I.M.Freedberg, A.Z.Eisen. Fitzpatrickís Dermatology in General Medicine. Vol. 4, 6th ed. New York: McGraw-Hill, Vol.7, PP.2119-30, 2358, 2575-80,2003.

[4] J.Sterling. Virus Infection. In Rooks Textbook of Dermatology. In: Burns T, Breathnach S, Cox N, 
Griffiths CAR editors.8th edition, Oxford Blackwell, Vol.2, PP.37-60,2004.

[5] P.Saitta, K.Krishnamurthy , L.H.Brown. Bleomycin in dermatology: A review of intralesional applications. Dermatol Surg , Vol. 34, PP.1299313,2008.

[6] E.Agius, J.M.Mooney, A.C.Bezzina . Dermojet delivery of bleomycin for the treatment of recalcitrant plantar warts. J Dermatolog. Treat, Vol. 17, PP.112-116,2006.

[7] D.R.McIlwain, T.Berger, T.W. Mak. Caspase functions in cell death and disease. Cold Spring Harbor perspectives in biology , Vol.5(4), PP.a008656,2013.

[8] T.Mesplede, D.Gagnon, F.Bergeron . degradation activity, expression, and subcellular localization of E6 proteins from 29 human papillomavirus genotypes. J. Virol, Vol. 86, PP.94-107,2012.

[9] M.D.Saber THESIS. Evaluation of the efficacy of intralesional bleomycin injection in treatment of warts histopathological and immunopathological study of bleomycin injection into skin;page 117,2005

[10] M.I.El-Ghareeb ， M.Khater, A.Alyaa . Autoinoculation versus Intralesional Injection of Bleomycin in theTreatment of Multiple Recalcitrant
Warts. Journal of Infectious Diseases and Therapy, Vol.6(5), PP.376-381,2018.

[11] S.B.Dhar, M.M.Rashid, A.Islam. Intralesional bleomycin in the treatment of cutaneous warts: A randomized clinical trial comparing it with cryotherapy ndian J Dermatol Venereol Leprol, Vol. 75 (3), PP.262-267,2009.

[12] Mahesh, vaibhav. Intralesional Bleomycin in theTreatment of common warts. Indian J Drugs Dermatol, Vol. 3, PP.73-77,2017.

[13] K.Tahir, A.Farhana , I.Usma. Efficacy and safety of intralesional 5-fluorouracil in treatment of warts. Journal of Pakistan Association of Dermatologists, Vol.28(3), PP.337-339, 2018.

[14] M.Kavya, B.M.Shashikumar, M.R.Harish .: Safety and Efficacy of Intralesional Vitamin D3 in Cutaneous Warts: An Open Uncontrolled Trial. J Cutan Aesthet Surg, Vol.10(2), PP.90-94,2017.

[15] K. Laura, V.Saggar, A.Akhavan . Intralesional bleomycin for warts: Patient satisfaction and treatment outcome. J Cutan Med Surg , Vol.19, PP.470-476,2015.

[16] S.Lippens, G.Denecker, P.Ovaere . Death penalty for keratinocytes: apoptosis versus cornification. Cell Death and Differentiation, Vol.12, PP.14971508,2005 